\title{
The current state of intermittent intraoperative neural monitoring for prevention of recurrent laryngeal nerve injury during thyroidectomy: a PRISMA-compliant systematic review of overlapping meta-analyses
}

\author{
Brandon Michael Henry ${ }^{1,2}$ - Matthew J. Graves ${ }^{1,2}$. Jens Vikse ${ }^{1,2,3}$ - Beatrice Sanna ${ }^{4}$. \\ Przemyslaw A. Pękala ${ }^{1,2}$. Jerzy A. Walocha ${ }^{1,2}$ - Marcin Barczyński ${ }^{5}$. \\ Krzysztof A. Tomaszewski ${ }^{1,2}$
}

Received: 15 February 2017 / Accepted: 26 March 2017 / Published online: 4 April 2017

(C) The Author(s) 2017. This article is published with open access at Springerlink.com

\begin{abstract}
Purpose Recurrent laryngeal nerve (RLN) injury is one of the most common and detrimental complications following thyroidectomy. Intermittent intraoperative nerve monitoring (IIONM) has been proposed to reduce prevalence of RLN injury following thyroidectomy and has gained increasing acceptance in recent years.

Methods A comprehensive database search was performed, and data from eligible meta-analyses meeting the inclusion criteria were extracted. Transient, permanent, and overall RLN injuries were the primary outcome measures. Quality assessment via AMSTAR, heterogeneity appraisal, and selection of best evidence was performed via a Jadad algorithm.
\end{abstract}

Brandon Michael Henry and Matthew J. Graves are equal contributors.

Electronic supplementary material The online version of this article (doi:10.1007/s00423-017-1580-y) contains supplementary material, which is available to authorized users.

Brandon Michael Henry

bmhenry55@gmail.com

1 International Evidence-Based Anatomy Working Group, 12 Kopernika St, 31-034 Krakow, Poland

2 Department of Anatomy, Jagiellonian University Medical College, 12 Kopernika St, 31-034 Krakow, Poland

3 Division of Medicine, Stavanger University Hospital, Gerd-Ragna Bloch Thorsens gate 8, 4011 Stavanger, Norway

4 Faculty of Medicine and Surgery, University of Cagliari, S.S. 554, Bivio Sestu, 09042 Monserrato, CA, Sardinia, Italy

5 Department of Endocrine Surgery, Third Chair of General Surgery, Jagiellonian University Medical College, 35-37 Pradnicka St, 31202 Krakow, Poland
Results Eight meta-analyses met the inclusion criteria. Metaanalyses included between 6 and 23 original studies each. Via utilization of the Jadad algorithm, the selection of best evidence resulted in choosing of Pisanu et al. (Surg Res 188:152161, 2014). Five out of eight meta-analyses demonstrated non-significant $(p>0.05)$ RLN injury reduction with the use of I-IONM versus nerve visualization alone.

Conclusions To date, I-IONM has not achieved a significant level of RLN injury reduction as shown by the meta-analysis conducted by Pisanu et al. (Surg Res 188:152-161, 2014). However, most recent developments of IONM technology including continuous vagal IONM and concept of staged thyroidectomy in case of loss of signal on the first side in order to prevent bilateral RLN injury may provide additional benefits which were out of the scope of this study and need to be assessed in further prospective multicenter trials.

Keywords Intermittent intraoperative nerve monitoring • Recurrent laryngeal nerve $\cdot$ Thyroidectomy $\cdot$ Vocal fold paresis $\cdot$ Iatrogenic injury

\section{Introduction}

Intermittent intraoperative nerve monitoring (I-IONM) made its debut in thyroid surgery in the late 1960s with promise to reduce procedure iatrogenic nerve injury [1]. I-IONM has gained popularity in recent years with ever increasing pressures on surgeons for complication-free procedures. Despite its increasing use, I-IONM is still presently considered an adjunctive tool during thyroid surgery, taking a secondary role behind the gold standard of direct recurrent laryngeal nerve (RLN) visualization [2]. Preoperative and postoperative 
laryngoscopic assessment of vocal cord function should also be appraised to determine baseline and postoperative function. This supplementary role of I-IONM is supported by the recommendations set forth by the German Association of Endocrine Surgeons' guidelines for thyroid disease and supported by the International Intraoperative Monitoring Study Group's international standards guideline statement $[2,3]$. It has also been proposed that I-IONM could play a more integral role in thyroid surgery during primary operations of highrisk patients, e.g., for retrosternal goiter, toxic goiter, Hashimoto's thyroiditis, and Graves' disease, or in patients undergoing revision surgery for recurrent goiter or local recurrence of thyroid cancer [4-6]. Various anatomic anomalies of the RLN such as extralaryngeal branching also pose a unique threat to the use of direct visualization and can potentially be more effectively identified with I-IONM [4]. Although many attempts have been made in recent years to statistically demonstrate the reliability of I-IONM as an essential tool for RLN identification, individual clinical studies have produced conflicting results and meta-analyses have yet to establish a uniformly acceptable conclusion. Several meta-analyses conducted in the last 5 years corroborate the current notion that IIONM should not be incorporated into the standard of care for thyroid surgery [7-11]. Three analyses conducted by Zheng et al., Yang et al., and Wong et al. demonstrated just the opposite, in that there were significant benefits of I-IONM use $[6,12,13]$. Individual studies such as Thomusch et al. [14] and Barczynski et al. [15] have also contributed to these conflicting results.

It has been noted that from a financial perspective, I-IONM does not become justifiably cost effective unless it is able to achieve a 50.4\% reduction in injuries compared to traditional direct visualization $[16,17]$. Additionally, it does not significantly reduce operative time [16, 17]. Results of statistically significant injury reduction have been largely mixed and inconsistent with no obvious trend supporting movement towards full-time I-IONM use [13] or I-IONM as purely adjunctive worth [7-10]. Determining whether an improvement in transient vocal fold palsy (VFP) alone or a reduction in permanent VFP is required for implementing widespread IIONM use is another point for debate.

A study by Sturgeon et al. indicated that approximately $37 \%$ of surgeons either routinely or in select cases use IIONM during thyroid procedures [18]. I-IONM use according to Sanabria et al. and Barczynski et al. is also stratified based on equipment availability, experience, and surgeon age [11, 19]. Injury to the RLN during thyroid operations is notably one of the most severe postoperative complications patients experience $[4,9]$. VFP is also the most frequent citing cause for litigation post thyroidectomy, as well as a significant detriment to patient quality of life [20]. Injuries bear a vast range of severity from unilateral transient VFP causing hoarseness to permanent bilateral VFP resulting in airway obstruction requiring tracheostomy [10]. Rates of transient VFP and permanent VFP have been reported as 9.8 and $2.3 \%$, respectively [21].

Technological advances in I-IONM are occurring and the time is likely approaching when I-IONM will become standard practice for thyroid surgery patients. Yarborough et al. cite that I-IONM can play a vital role in three ways for surgeons: substantiating decisions in cases of aberrant anatomy and pathology, routine intraoperative RLN identification, and assessing postoperative RLN function [22]. Preserving RLN function is a top priority, and affording surgeons the proper information and opportunity to use all available technology to lower the injury rate is paramount. The aim of our review is to directly compare the previously conducted meta-analyses on the use of I-IONM versus direct RLN visualization by assessing rates of VFP. The ultimate goal of this analysis is to provide clarification of the differing conclusions about IIONM use that have been presented in literature to date and put them in a perspective of rapidly developing innovations like continuous IONM technology or concept of staged thyroidectomy previously not evaluated in any of the meta-analyses. We hope that this review will serve to better guide the standards of clinical therapy and the use of I-IONM in future operative procedures.

\section{Methods}

\section{Search strategy}

Through February 2017, a database search was performed through PubMed, ScienceDirect, EMBASE, BIOSIS, SciELO, Web of Science, and Cochrane Library in order to identify eligible articles for the review. The search strategy employed for PubMed is presented in Table 1. No date limits or language restrictions were applied. The references in the included articles were also extensively searched. The Preferred Reporting Items for Systematic Reviews and Meta-Analyses (PRISMA) guidelines were strictly followed throughout this systematic review (Supplemental Item 1) [23].

\section{Study selection criteria}

Studies were deemed eligible for inclusion if they were a meta-analysis of randomized control trials or observational studies (prospective or retrospective) comparing I-IONM to direct visualization of the RLNs during thyroidectomy with data reporting incidence of any type of VFP. The exclusion criteria included (1) systematic reviews not conducting metaanalysis or pooling of the data; (2) meta-analysis reporting incomplete data; and (3) conference abstracts, narrative reviews, commentary, or non-peer reviewed publications. 
Table 1 Search strategy for PubMed

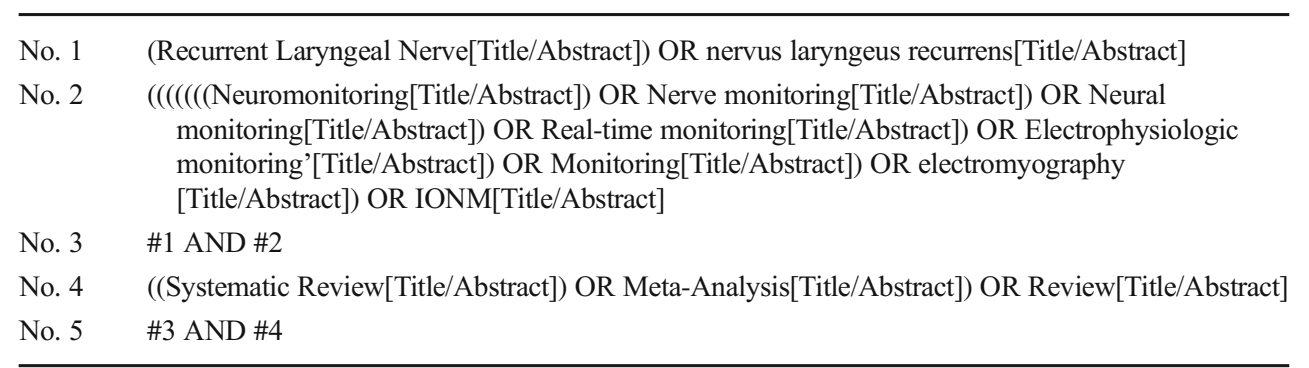

\section{Eligibility assessment}

All studies were independently assessed for eligibility by two reviewers (B.M.H \& J.V.). Any disparities arising during the assessment were resolved by a consensus among all the reviewers, after consulting with the authors of the original study, if possible. All full-text articles published in languages not spoken fluently by the authors were translated for further eligibility assessment by medical professionals fluent in both English and the original language of the manuscript.

\section{Data extraction}

Data were independently extracted from the included analyses by three independent reviewers (J.V., P.P., B.S.). Basic data included demographic information such as year, country, studies included in their analysis, databases searched, and study design. The primary outcomes examined in this systematic review were the incidence of transient, persistent, and overall RLN injury.

\section{Quality assessment}

Quality assessment of the included studies was conducted by two independent reviewers (J.V., B.M.H.). Any disagreements were resolved by discussion until mutual consensus or involving a third reviewer (M.J.G.). The Assessment of Multiple Systematic Reviews (AMSTAR) method was used for study appraisal [24]. The AMSTAR performed included 11 criteria and has become the standard for meta-analysis and systematic review quality assessment [25].

\section{Selection of best evidence}

Evaluation of included meta-analyses was performed through the implementation of a Jadad decision algorithm [26]. Utilization of the Jadad algorithm allowed for the discernment between varying methodological practices in different metaanalyses and systematic reviews ranging from data-extraction, inclusion/exclusion criteria, to statistical analyses performed [26]. The Jadad system is designed to allow for a clear assessment of the evidence presented in a given analysis and allows for the determination of which study provides the best overall evidence given the present information.

\section{Results}

\section{Study identification and characteristics of included studies}

A summary of the flow of studies through the systematic review is presented in Fig. 1. A search through the major electronic databases identified 476 articles; none were identified when the references of the included studies were searched. After 151 duplicates had been excluded and 325 records screened, 22 articles were further assessed for eligibility by full text. Among these, 14 were excluded and 8 were included in the review. One meta-analysis by Rulli et al. [27] was excluded due to incomplete data with respect to details of their samples.

The characteristics of the meta-analyses included in this study are detailed in Table 2. Included studies ranged in time from 2011 to 2017 and included between 6 and 23 studies in their analyses. Table 3 provides cross-linking of the original studies included in the 8 meta-analyses reviewed in this present work. The original studies included in the different meta-analyses varied significantly despite the research largely targeting the same primary outcome. Additionally, the meta-analysis conducted by Wong et al. [6] focused entirely on procedures which were deemed "highrisk," such as those undergoing repeat operation and operations due to significant pathology.

\section{Search methodology}

Of the eight studies included, they differed significantly in their literature search methodology and the databases in which they included. The databases utilized by each included metaanalysis are detailed in Table 4. All studies included a literature search of MEDLINE; however, all other databases were not universally searched among the included studies.

\section{AMSTAR quality assessment}

The results of the AMSTAR checklist are presented in detail in Table 5. Studies uniformly developed study designs prior to 


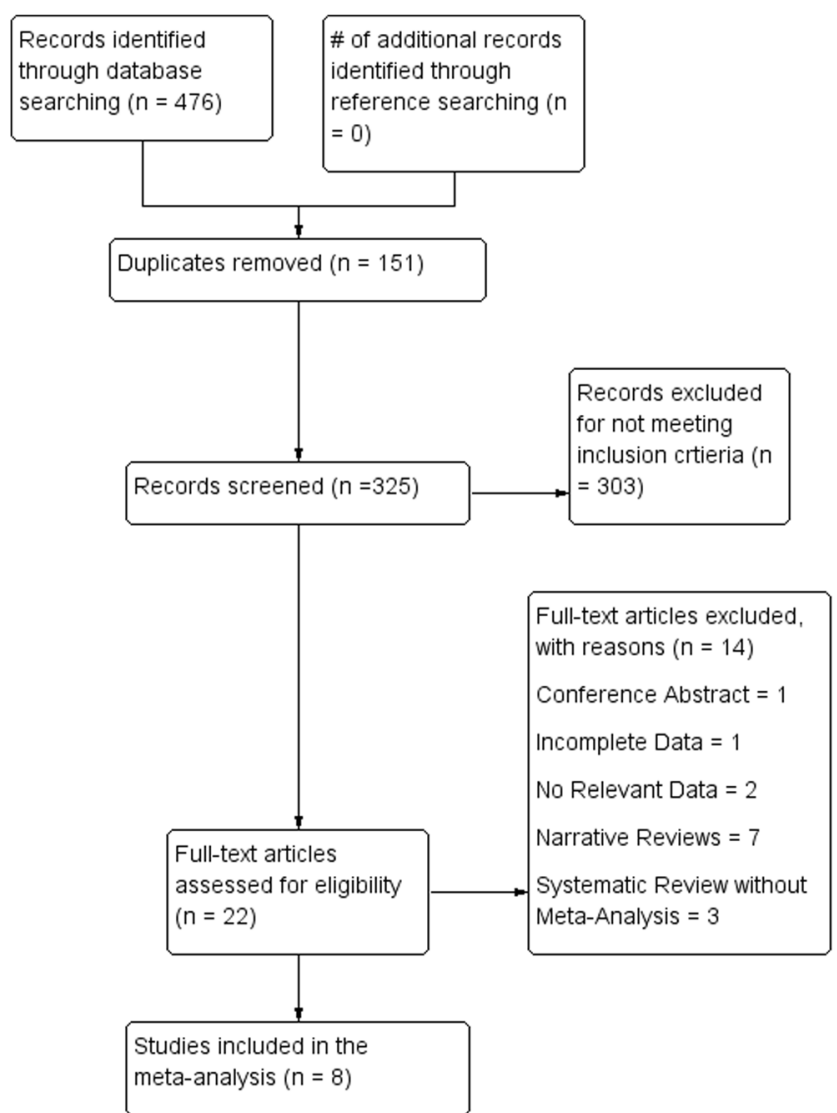

Fig. 1 PRISMA flowchart of study identification and inclusion in the meta-analysis

execution as well as performed systematic literature searches of their intended databases. Quality assessment was largely overlooked in many cases and rarely did quality factor into decision-making when it came to conclusions.

\section{Overview of primary and secondary outcomes}

The results of the three primary outcomes, overall, transient, and persistent RLN injury, are summarized in Tables 6, 7, and 8 , respectively.

\section{Heterogeneity assessment}

Heterogeneity of the primary outcomes studied in this analysis was extracted and recorded. A detailed breakdown of heterogeneity data is available in Table 9. The $I^{2}$ statistic was utilized in 6/8 [9-11, 13, 12, 6] included studies and was calculable in a fifth [8]. No heterogeneity was recorded by Malik et al. [7].

\section{Results of Jadad decision algorithm}

Included meta-analyses were analyzed for methodological quality using a Jadad algorithm (Fig. 2). The Jadad assessed quality based on their utilization of the same primary outcome, studies included, and selection criteria. Furthermore, studies were compared on their use of included study quality in drawing conclusions, language restrictions present, and data analysis procedures. As a result, the meta-analysis with the highest quality was selected. Pisanu et al. [8] was selected and supports the notion that there is no statistically significant reduction in RLN injury between procedures with I-IONM over direct RLN visualization.

\section{Discussion}

This comprehensive review is aimed at comparing and summarizing meta-analyses on the rates of RLN injury between groups undergoing either direct visualization or I-IONM during

Table 2 Characteristics of included studies

\begin{tabular}{|c|c|c|c|c|c|c|c|c|c|}
\hline \multirow[t]{3}{*}{ Study ID } & \multirow[t]{3}{*}{ Journal } & \multirow{3}{*}{$\begin{array}{l}\text { Literature search } \\
\text { valid through }\end{array}$} & \multirow[t]{3}{*}{ Published } & \multicolumn{5}{|c|}{ Number of included studies } & \multirow[t]{3}{*}{ Case series } \\
\hline & & & & \multirow[t]{2}{*}{ Total } & \multirow[t]{2}{*}{ RCT } & \multicolumn{3}{|c|}{ Comparative } & \\
\hline & & & & & & Total & Prospective & Retrospective & \\
\hline Higgins 2011 & The Laryngoscope & July 2008 & 2011 & $9(+34)$ & 1 & 8 & 4 & 4 & 34 \\
\hline Lombardi 2016 & Surgery & August 2014 & June 2016 & 14 & 4 & 10 & 5 & 5 & 0 \\
\hline Malik 2016 & World J Surg & Unknown & June 2016 & $15(+2)$ & 1 & 14 & Unknown & Unknown & 2 \\
\hline Pisanu 2014 & J Surgical Research & August 2013 & 2014 & 20 & 3 & 17 & 7 & 10 & 0 \\
\hline Sanabria 2013 & Eur Arch Otorhinolaryngol & December 2012 & May 2013 & 6 & 6 & 0 & 0 & 0 & 0 \\
\hline Zheng 2013 & J Formos Med Assoc & April 2011 & 2013 & 14 & 2 & 12 & 8 & 4 & 0 \\
\hline Yang 2017 & International J Surgery & July 2016 & 2017 & 23 & 4 & 19 & 6 & 13 & 0 \\
\hline Wong $2017^{\mathrm{a}}$ & International J Surgery & September 2015 & 2017 & 10 & 0 & 10 & 2 & 8 & 0 \\
\hline
\end{tabular}

$R C T$ randomized controlled trial

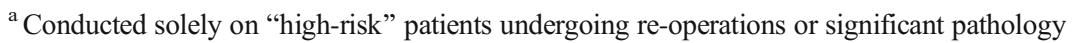


Table 3 Citation matrix of primary studies included in previous meta-analyses (excluding case series)

\begin{tabular}{|c|c|c|c|c|c|c|c|c|}
\hline & Higgins 2011 & Lombardi 2016 & Malik 2016 & Pisanu 2014 & Sanabria 2013 & Zheng 2013 & Yang 2017 & Wong 2017 \\
\hline Agha 2008 & - & & & - & & - & - & \\
\hline Alesina 2012 & & - & & - & & & - & - \\
\hline Atallah 2009 & & & & - & & & & - \\
\hline Barczynski 2009 & - & - & - & - & - & - & - & \\
\hline Barczynski 2011 & & & & - & & & & - \\
\hline Barczynski 2012 & & - & & & - & & & \\
\hline Barczynski 2014 & & - & & & & & - & - \\
\hline Brauckhoff 2002 & & & & & & - & & \\
\hline Calo 2013 & & & & - & & & & \\
\hline Calo 2014 & & - & & & & & & - \\
\hline Calo 2016 & & & & & & & - & \\
\hline Chan 2006 & - & - & - & - & & - & - & - \\
\hline Chiang 2008 & & & - & & & & - & \\
\hline Danschutter 2015 & & & & & & & - & \\
\hline De Falco 2014 & & - & & & & & & \\
\hline Diongi 2009 & & - & & - & - & & - & \\
\hline Dralle 2004 & - & - & - & - & & - & & - \\
\hline Duclos 2011 & & & - & - & & & - & \\
\hline Frattini 2010 & & & - & - & & - & - & - \\
\hline Gremillion 2012 & & & & - & & & - & \\
\hline Hei 2016 & & & & & & & - & \\
\hline Khaled 2012 & & & & & - & & & \\
\hline Lifante 2009 & & & & & - & & & \\
\hline Netto 2007 & - & & - & - & & - & - & \\
\hline Page 2015 & & & & & & & - & \\
\hline Prokopakis 2013 & & & & & & & & - \\
\hline Robertson 2004 & - & & - & - & & - & - & \\
\hline San 2010 & & & & & & - & & \\
\hline Sari 2010 & & - & & - & - & - & - & \\
\hline Shindo 2007 & - & & & - & & & - & \\
\hline Snyder 2013 & & & - & & & & & \\
\hline Stevens 2012 & & - & & - & & & - & \\
\hline Terris 2007 & - & - & - & - & & - & - & \\
\hline Thomusch 2002 & & - & - & & & - & & \\
\hline Witt 2005 & - & - & & - & & - & - & \\
\hline Xie 2016 & & & & & & & - & \\
\hline Yarbrough 2004 & & & - & - & & - & - & - \\
\hline
\end{tabular}

thyroidectomy. The meta-analysis conducted by Pisanu et al. was determined to be of the highest methodological quality when compared to the other included studies [8]. The evidence to date supports the notion that I-IONM does not provide for significant reduction in postoperative RLN injury challenging rationale for its widespread implementation [8].

Meta-analyses have long been utilized to increase the statistical power through the combination of smaller studies in hopes of uncovering meaningful data for use in clinical practice. In the case of I-IONM, the recommendations have varied widely between individual studies and in the several analyses conducted in recent years. With the continually variable conclusions being published on the subject, it becomes very difficult for clinicians and scientists alike to form meaningful practice changing policy. It is the intent of this analysis to provide a pathway for these individuals and agencies to easily navigate these conflicting studies.

In 1997, Jadad et al. devised a system with which to sift through these analyses that have come to differing conclusions [26]. It was proposed that there are several sources that could 
Table 4 Databases searched in each original meta-analysis

\begin{tabular}{|c|c|c|c|c|c|c|c|c|}
\hline Databases & $\begin{array}{l}\text { Higgins } \\
2011\end{array}$ & $\begin{array}{l}\text { Lombardi } \\
2016\end{array}$ & $\begin{array}{l}\text { Malik } \\
2016\end{array}$ & $\begin{array}{l}\text { Pisanu } \\
2014\end{array}$ & $\begin{array}{l}\text { Sanabria } \\
2013\end{array}$ & $\begin{array}{l}\text { Zheng } \\
2013\end{array}$ & $\begin{array}{l}\text { Yang } \\
2017\end{array}$ & $\begin{array}{l}\text { Wong } \\
2017\end{array}$ \\
\hline MEDLINE & - & - & - & - & - & - & - & - \\
\hline EMBASE & - & & - & - & - & - & - & - \\
\hline Cochrane & - & - & & - & - & - & - & - \\
\hline clinicaltrials.gov & - & & & & & & & \\
\hline $\begin{array}{l}\text { The National } \\
\text { Guideline } \\
\text { Clearinghouse }\end{array}$ & - & & & & & & & \\
\hline Scopus & & - & & & & & & \\
\hline Google Scholar & & & & - & & & & \\
\hline \multicolumn{9}{|l|}{ Ovid } \\
\hline LILACS & & & & & - & & & \\
\hline
\end{tabular}

lead to these discordant results such as inclusion criteria, extraction techniques, statistical analysis procedures, and quality assessment [26].

The meta-analysis conducted by Pisanu et al. was the study selected that best reflects the present literature [8]. It can be noted in Table 3 that Pisanu et al. included the second highest number of studies in their analysis [8]. The chosen study achieved a score of 6 in the AMSTAR checklist utilized. The score was largely lowered by the lack of quality assessment of the studies that were included. There were five studies which resulted in an AMSTAR of higher value: Higgins et al. [10], Sanabria et al. [11], Zheng et al. [13], Yang et al. [12], and Wong et al. [6]. Through the utilization of the Jadad method, and assessing the number of studies included, the databases searched, journal of publication, and methodology, the authors still deemed Pisanu et al. to be the meta-analysis of highest quality [8]. The conclusion that I-IONM should be used as a purely adjunctive measure that resulted in Pisanu et al. $(p=0.471)$ [8] was largely supported by the other analyses included in this present review [Malik $2016(p>0.05)$ [7], Lombardi $2016(p>0.05)$ [9], Higgins $2011(p=0.11)$ [10], Sanabria $2013(p=0.15)$ [11]], clashing with the results of three reviews (Zheng 2013, $p=0.007$, Yang 2017, $p=0.041$, Wong 2017, $p=0.003,0.021$, and 0.05) [6, 12, 13]. The meta-analysis conducted by Wong et al. [6] demonstrated significant rates of injury reduction in both overall and transient VFP; it should however be noted that this study focused entirely on high-risk procedures of patients undergoing re-operation or those with significant pathology such as malignancy or retrosternal goiter.

Table 5 AMSTAR Criteria for included meta-analyses

\begin{tabular}{|c|c|c|c|c|c|c|c|c|}
\hline Items & $\begin{array}{l}\text { Higgins } \\
2011\end{array}$ & $\begin{array}{l}\text { Lombardi } \\
2016\end{array}$ & $\begin{array}{l}\text { Malik } \\
2016\end{array}$ & $\begin{array}{l}\text { Pisanu } \\
2014\end{array}$ & $\begin{array}{l}\text { Sanabria } \\
2013\end{array}$ & $\begin{array}{l}\text { Zheng } \\
2013\end{array}$ & $\begin{array}{l}\text { Yang } \\
2017\end{array}$ & $\begin{array}{l}\text { Wong } \\
2017\end{array}$ \\
\hline Was an "a priori" design provided? & 1 & 1 & 1 & 1 & 1 & 1 & 1 & 1 \\
\hline Was there duplicate selection and data extraction? & 1 & 1 & 0 & 1 & 1 & 1 & 1 & 1 \\
\hline Was a comprehensive literature search performed? & 1 & 1 & 1 & 1 & 1 & 1 & 1 & 1 \\
\hline $\begin{array}{l}\text { Was the status of publication used as an inclusion } \\
\text { criterion? }\end{array}$ & 0 & 0 & 0 & 0 & 0 & 1 & 0 & 0 \\
\hline Was a list of included/excluded studies provided? & 0 & 0 & 0 & 0 & 0 & 0 & 0 & 0 \\
\hline Were the profiles of the included studies provided? & 1 & 1 & 0 & 1 & 1 & 1 & 1 & 1 \\
\hline $\begin{array}{l}\text { Was the methodological quality of the included studies } \\
\text { evaluated and documented? }\end{array}$ & 0 & 1 & 0 & 0 & 1 & 1 & 0 & 1 \\
\hline $\begin{array}{l}\text { Was the specific quality of the included studies used } \\
\text { appropriately in formulating conclusions? }\end{array}$ & 0 & 0 & 0 & 0 & 1 & 0 & 0 & 0 \\
\hline $\begin{array}{l}\text { Were the methods used to combine the findings of } \\
\text { studies appropriate? }\end{array}$ & 1 & 1 & 0 & 1 & 1 & 1 & 1 & 1 \\
\hline Was the publication bias evaluated? & 1 & 0 & 0 & 0 & 1 & 1 & 1 & 1 \\
\hline Were the conflicts of interest stated? & 1 & 0 & 0 & 1 & 0 & 0 & 1 & 1 \\
\hline Total score & 7 & 6 & 2 & 6 & 8 & 8 & 7 & 8 \\
\hline
\end{tabular}


Table 6 Overall incidence of vocal fold palsy between IONM and direct visualization

\begin{tabular}{|c|c|c|c|c|c|c|c|c|}
\hline & \multicolumn{8}{|c|}{ Overall VFP (overall incidence) } \\
\hline & \multicolumn{3}{|l|}{ IONM } & \multicolumn{3}{|c|}{ Visual identification only } & \multicolumn{2}{|l|}{ Odds ratios } \\
\hline & $n=($ nerves $)$ & VFP & $\%$ & $n=($ nerves $)$ & VFP & $\%$ & OR $(95 \% \mathrm{CI})$ & $p$ value \\
\hline Higgins 2011 (excluding case series) & 20,500 & 729 & 3.56 & 7939 & 325 & 4.09 & $0.87(0.73-1.03)$ & 0.11 \\
\hline Higgins 2011 (case series) & 7435 & 253 & 3.4 & 29,440 & 845 & 2.87 & $1.32(0.77-2.27)$ & 0.31 \\
\hline Malik 2016 & 25,843 & 822 & 3.18 & 18,732 & 718 & 3.83 & NR & - \\
\hline Pisanu 2014 & 24,038 & 834 & 3.47 & 11,475 & 421 & 3.67 & $0.94(0.83-1.06)$ & 0.471 \\
\hline Sanabria 2013 & 1446 & 40 & 2.77 & 1466 & 70 & 4.77 & NR & - \\
\hline Zheng 2013 & 23,298 & 786 & 3.37 & 12,898 & 485 & 3.76 & $0.74(0.59-0.92)$ & 0.007 \\
\hline Yang 2017 & 8668 & 273 & 3.15 & 8535 & 373 & 4.37 & $0.81(0.66-0.99)$ & 0.041 \\
\hline Wong 2017 (high-risk thyroidectomy) & 6155 & 151 & 2.5 & 4460 & 201 & 4.5 & $1.42(1.12-1.79)$ & 0.003 \\
\hline Wong 2017 (re-operation thyroidectomy) & 1751 & 78 & 4.45 & 1497 & 114 & 7.61 & $1.48(1.06-2.06)$ & 0.021 \\
\hline Wong 2017 (thyroidectomy for malignancy) & 2468 & 52 & 2.11 & 1596 & 55 & 3.45 & $1.52(1.00-2.31)$ & 0.05 \\
\hline
\end{tabular}

$I O N M$ intraoperative nerve monitoring, $V F P$ vocal fold palsy, $O R$ odds ratio, $N R$ not reported

I-IONM in theory appears as an ideal tool that could provide better outcomes for patients. It has been noted that with traditional intermittent (non-continuous) IONM techniques, injuries are often discovered by the machine but only after they have already occurred [28]. Until injuries can be prevented as opposed to being simply identified, this approach does not seem to be justifiable. However, results reported by Bergenfelz et al. in a database from a Swedish multicenter audit comprising 3660 patients undergoing thyroid surgery showed that RLN injury was recognized intraoperatively in only $16(11.3 \%)$ out of 142 patients with a damaged nerve [29]. This in agreement with recent studies showing that RLN injury most often occurs to a visually observed nerve. Hence, I-IONM has a potential to improve the intraoperative
RLN management by elucidating mechanisms of nerve injury. In addition, I-IONM is an effective tool in staging planned bilateral thyroid surgeries in cases of intraoperative RLN injury on the side of initial dissection [30]. This issue is of great importance in prevention of bilateral RLN injury which occurs in approximately $0.2 \%$ of patients undergoing thyroidectomy leading to significant deterioration of quality of life and medicolegal claims [31]. Bergenfelz et al. analyzed the risk of RLN palsy in a cohort of 5252 patients undergoing thyroidectomy with and without intraoperative nerve monitoring who were registered in the Scandinavian Quality Register for Thyroid, Parathyroid and Adrenal Surgery in 2009-2013 [32]. I-IONM was used in 3277 operations (62.4\%), and postoperative laryngoscopy was performed in 1757 patients

Table 7 Incidence of transient vocal fold palsy between IONM and direct visualization

\begin{tabular}{|c|c|c|c|c|c|c|c|c|}
\hline & \multicolumn{8}{|c|}{ Transient VFP } \\
\hline & \multicolumn{3}{|l|}{ IONM } & \multicolumn{3}{|c|}{ Visual identification only } & \multicolumn{2}{|l|}{ Odds ratios } \\
\hline & $n=($ nerves $)$ & Trans. VFP & $\%$ & $n=($ nerves $)$ & Trans. VFP & $\%$ & OR $(95 \% \mathrm{CI})$ & $p$ value \\
\hline Higgins 2011 (excluding case series) & 20,500 & 552 & 2.69 & 7939 & 234 & 2.95 & $0.94(0.80-1.10)$ & .44 \\
\hline Higgins 2011(case series) & 7435 & 213 & 2.86 & 29,440 & 697 & 2.37 & $1.43(0.86-2.38)$ & .16 \\
\hline Pisanu 2014 & 24,038 & 630 & 2.62 & 11,475 & 312 & 2.72 & $0.95(0.82-1.10)$ & 0.552 \\
\hline Sanabria 2013 & 1446 & 32 & 2.21 & 1466 & 58 & 3.96 & NR & - \\
\hline Zheng 2013 & 23,298 & 596 & 2.56 & 12,898 & 350 & 2.71 & $0.80(0.65-0.99)$ & .04 \\
\hline Yang 2017 & 8668 & 158 & 1.82 & 8535 & 220 & 2.58 & $0.76(0.61-0.94)$ & 0.013 \\
\hline Wong 2017 (high-risk thyroidectomy) & 3017 & 71 & 2.5 & 3332 & 129 & 3.9 & $1.47(1.07-2.00)$ & 0.016 \\
\hline Wong 2017 (re-operation thyroidectomy) & 812 & 35 & 4.31 & 1188 & 75 & 6.31 & $1.49(0.95-2.33)$ & 0.082 \\
\hline Wong 2017 (thyroidectomy for malignancy) & 1282 & 21 & 1.64 & 1160 & 36 & 3.1 & $1.90(1.08-3.35)$ & 0.026 \\
\hline
\end{tabular}

$I O N M$ intraoperative nerve monitoring, $V F P$ vocal fold palsy, $O R$ odds ratio, $N R$ not reported 
Table 8 Incidence of persistent vocal fold palsy between IONM and direct visualization

\begin{tabular}{|c|c|c|c|c|c|c|c|c|}
\hline & \multicolumn{8}{|c|}{ Persistent VFP } \\
\hline & \multicolumn{3}{|l|}{ IONM } & \multicolumn{3}{|c|}{ Visual identification only } & \multicolumn{2}{|l|}{ Odds ratios } \\
\hline & $n=($ nerves $)$ & Pers. VFP & $\%$ & $n=($ nerves $)$ & Pers. VFP & $\%$ & OR $(95 \% \mathrm{CI})$ & $p$ value \\
\hline Higgins 2011 (excluding case series) & 20,500 & 167 & 0.81 & 7939 & 79 & 0.99 & $0.88(0.66-1.16)$ & .36 \\
\hline Higgins 2011(case series) & 7435 & 42 & 0.56 & 29,440 & 146 & 0.5 & $0.95(0.43-2.10)$ & .9 \\
\hline Lombardi 2016 (NRS; 6 months) & 21,197 & 158 & 0.75 & 11,093 & 94 & 0.85 & NR & - \\
\hline Lombardi 2016 (NRS; 12 months) & 3152 & 21 & 0.67 & 3378 & 35 & 1.03 & NR & - \\
\hline Lombardi 2016 (NRS; overall) & 24,349 & 179 & 0.73 & 14,471 & 129 & 0.89 & NR & - \\
\hline Lombardi 2016 (RCTs) & 1465 & 8 & 0.55 & 1458 & 12 & 0.82 & NR & - \\
\hline Pisanu 2014 & 24,038 & 190 & 0.79 & 11,475 & 106 & 0.92 & $0.88(0.69-1.14)$ & 1 \\
\hline Sanabria 2013 & 1446 & 8 & 0.55 & 1466 & 12 & 0.82 & NR & - \\
\hline Zheng 2013 & 23,508 & 183 & 0.78 & 13,097 & 126 & 0.96 & $0.80(0.62-1.03)$ & .09 \\
\hline Yang 2017 & 8668 & 58 & 0.67 & 8535 & 91 & 1.06 & $0.78(0.55-1.09)$ & 0.146 \\
\hline Wong 2017 (high-risk thyroidectomy) & 6095 & 80 & 1.31 & 4399 & 72 & 1.64 & $1.33(0.94-1.88)$ & 0.104 \\
\hline Wong 2017 (re-operation thyroidectomy) & 1691 & 43 & 2.54 & 1436 & 39 & 2.72 & $1.40(0.87-2.27)$ & 0.171 \\
\hline Wong 2017 (thyroidectomy for malignancy) & 2468 & 31 & 1.26 & 1596 & 19 & 1.19 & $1.13(0.61-1.11)$ & 0.696 \\
\hline
\end{tabular}

$I O N M$ intraoperative nerve monitoring, $V F P$ vocal fold palsy, $O R$ odds ratio, $R C T$ randomized controlled trial, $N R S$ non-randomized studies, $N R$ not reported

(33.5\%) [32]. Early VFP occurred in 217 patients (4.1\%), of which 3 were bilateral, all in the group without I-IONM. Permanent VFP occurred in 62 patients (1.2\%). In the multivariable analysis of 1757 patients who had postoperative laryngoscopy, the use of I-IONM was not associated with a decreased risk of early VFP [OR 0.67 (95\% CI 0.44-1.01)], but decreased the risk of permanent VFP [OR 0.43 (95\% CI 0.190.93)]. Thus, data arising from a prospective register reflect to a greater extent some details of current surgical practice landscape in thyroid surgery rather than data from meta-analysis based on mixed and often inclusive of poorly reported retrospective case series.

It was noted by Chan et al. that among a survey of members of the American Association of Endocrine Surgeons, protection from litigation was cited as a primary reason for I-IONM use [33]. The addition of costly procedures to standard operative technique can only be rationalized by an improvement in clinical outcomes and should not be on the basis of legal protection [16]. The use of I-IONM is also associated with a learning curve and is predominantly utilized by surgeons under the age of $40[11,19]$. This learning curve can be associated with a period of higher incidence of injury which should be factored into research conducted in the future [7, 34]. Hence, training and exposure to the standardized utilization of I-IONM technique during thyroid surgery by attending to one of the hands-on courses organized worldwide and accredited by the International Neural Monitoring Study Group in Thyroid and Parathyroid Surgery should be warranted to optimize the clinical benefit. Finally, the most recent developments of IONM technology including continuous vagal IONM with intraoperative real-time electromyography of the vocalis muscles and its potential to recognize the imminent RLN injury which could be prevented by modifying surgical maneuvers seem to be a very promising tool considered to be a
Table 9 Heterogeneity $\left(I^{2}\right.$ statistic) of primary outcomes included in the meta-analysis

\begin{tabular}{cllllllll}
\hline & Higgins & Lombardi & Malik & Pisanu & Sanabria & Zheng & Yang & Wong \\
& 2011 & 2016 & 2014 & 2013 & 2017 & 2017 \\
\hline $\begin{array}{c}\text { Overall } \\
\text { VFP }\end{array}$ & $39 \%$ & $0 \%$ & - & $0 \%$ & - & $33 \%$ & $21 \%$ & $6.3 \%$ \\
$\begin{array}{c}\text { Transient } \\
\text { VFP } \\
\begin{array}{c}\text { Persistent } \\
\text { VFP }\end{array}\end{array}$ & $0 \%$ & $0 \%$ & - & $0 \%$ & $31 \%$ & $15 \%$ & $0 \%$ & $6.3 \%$ \\
\hline
\end{tabular}

- no heterogeneity data reported or calculable, VFP vocal fold palsy 


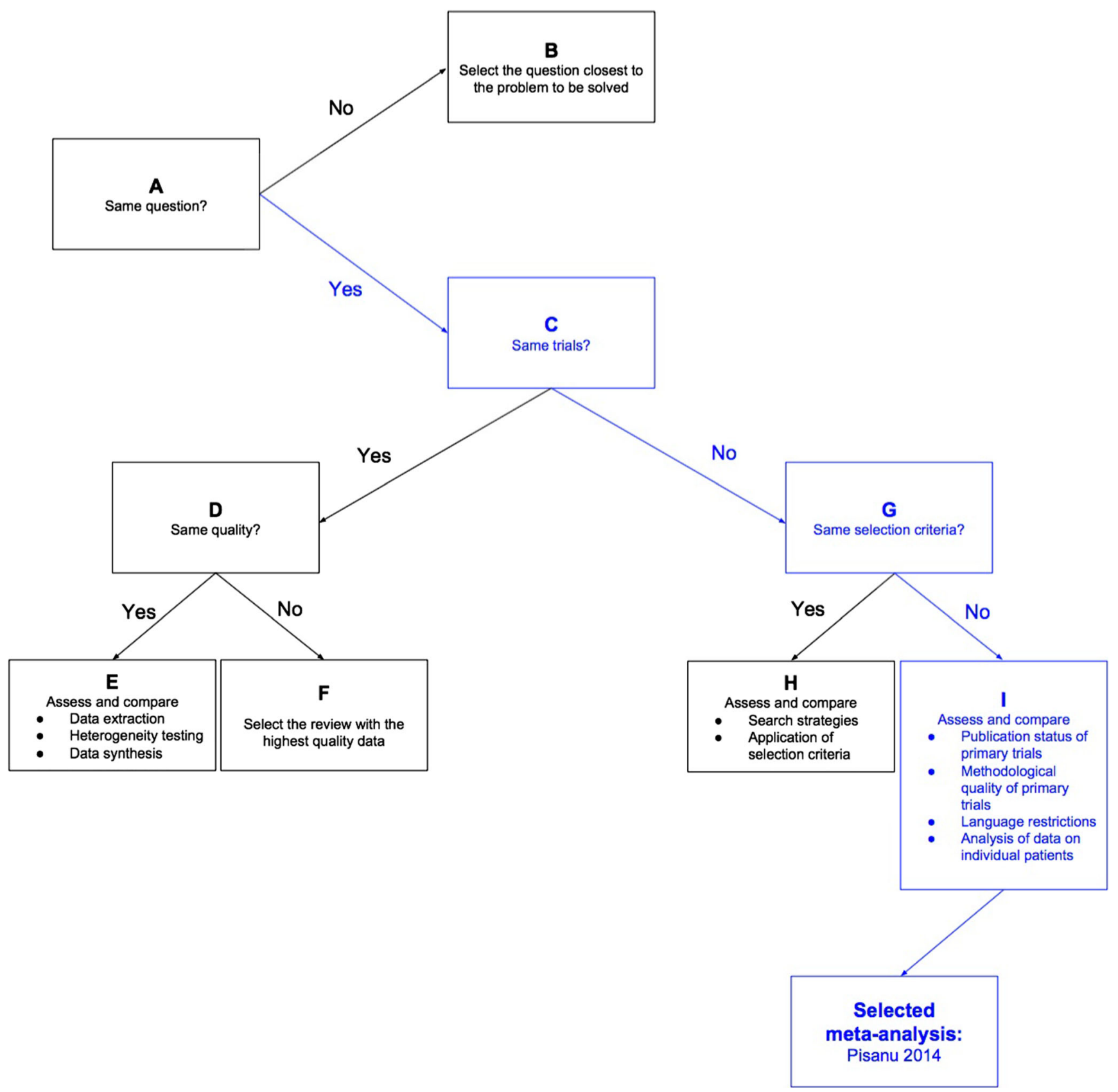

Fig. 2 Jadad decision algorithm

quantum leap forward in the prevention of the RLN injury during thyroid surgery [35]. It should be underlined that any of the published meta-analyses included continuous IONM studies as they were undertaken and published later on.

This systematic review was limited by a number of factors. Broadly speaking, the progress of IONM technology and the methods utilized varied in the included meta-analyses. It is likely that as IONM technology improves over time, there will be increasing utility for not only identifying the RLN but also preventing the RLN injury. Older studies may introduce a level of bias into meta-analyses because of the falsely depressed levels of RLN identification and non-standardized use of the I-IONM technique both for nerve identification and for prognostication of neural function. In addition, postoperative laryngoscopy was used on a select basis in many centers leading to underestimation of the true prevalence of the RLN injury. It is important in future investigations to reduce this potential bias through subgroup analysis on the basis of study year, use of the standardized approach to IONM, and postoperative laryngoscopy, as well as potentially the equipment used. With regard to the varying methodology, studies varied greatly on their search strategies, many of which did not 
include foreign language articles. Future meta-analysis on this topic should use a more rigorous methodology. Numerous issues with the originally included primary studies such as bias, study design, and sample heterogeneity may have had significant impacts on the final interpretations of their results.

There is a need for continuing investigation into the use of IONM as a primary method for RLN identification and prevention of postoperative complications. IONM is a developing technology, and likely with improvements, there will come a time where it does provide the necessary reduction in injuries to warrant its widespread implementation [7]. Additionally, original studies and new meta-analyses are needed to further investigate the use of IONM specifically in cases of reoperation as has been initiated by Wong et al. [6]. Many studies have shown insignificant improvement in postoperative complications in primary procedures, while demonstrating potential use in secondary operations $[7,33,36]$.

\section{Conclusion}

To date, I-IONM has not achieved a significant level of RLN injury reduction as shown by the meta-analysis conducted by Pisanu et al. [8]. However, most recent developments of IONM technology including continuous vagal IONM and concept of staged thyroidectomy in case of loss of signal on the first side in order to prevent bilateral RLN injury may provide additional benefits which were out of the scope of this study and need to be assessed in further prospective multicenter trials.

Acknowledgements Krzysztof A. Tomaszewski was supported by the Foundation for Polish Science (FNP) and by the Polish Ministry of Higher Education grant for young scientists.

Author contributions Study conception and design: BMH, MJG, JV, KAT; acquisition of data: JV, BS, PAP; analysis and interpretation of data: $\mathrm{BMH}, \mathrm{MJG}, \mathrm{JV}$, PAP; drafting of manuscript: BMH, MJG, PAP, MB, KAT; critical revision of manuscript: JAW, MB, KAT.

Compliance with ethical standards This article does not contain any studies with human participants or animals performed by any of the authors.

Conflict of interest The authors declare that there are no conflicts of interest.

Funding This study was supported by the statutory funding of Jagiellonian University Medical College.

Open Access This article is distributed under the terms of the Creative Commons Attribution 4.0 International License (http:// creativecommons.org/licenses/by/4.0/), which permits unrestricted use, distribution, and reproduction in any medium, provided you give appropriate credit to the original author(s) and the source, provide a link to the Creative Commons license, and indicate if changes were made.

\section{References}

1. Flisberg K, Lindholm T (1970) Electrical stimulation of the human recurrent laryngeal nerve during thyroid operation. Acta Otolaryngol 69:63-67. doi:10.3109/00016487009131523

2. Musholt TJ, Clerici T, Dralle H et al (2011) German Association of Endocrine Surgeons practice guidelines for the surgical treatment of benign thyroid disease. Langenbeck's Arch Surg 396:639-649. doi: 10.1007/s00423-011-0774-y

3. Randolph GW, Dralle H, Abdullah H et al (2011) Electrophysiologic recurrent laryngeal nerve monitoring during thyroid and parathyroid surgery: international standards guideline statement. Laryngoscope 121:S1-S16. doi:10.1002/lary.21119

4. Henry BM, Vikse J, Graves MJ et al (2016) Extralaryngeal branching of the recurrent laryngeal nerve: a meta-analysis of 28 , 387 nerves. Langenbeck's Arch Surg 401:913-923. doi:10.1007/ s00423-016-1455-7

5. Vasileiadis I, Karatzas T, Charitoudis G et al (2016) Association of Intraoperative Neuromonitoring with Reduced Recurrent Laryngeal Nerve Injury in Patients Undergoing Total Thyroidectomy. JAMA Otolaryngol Neck Surg 142:994. doi:10.1001/jamaoto.2016.1954

6. Wong KP, Mak KL, Wong CKH, Lang BHH (2017) Systematic review and meta-analysis on intra-operative neuro-monitoring in high-risk thyroidectomy. Int J Surg 38:21-30. doi:10.1016/j.ijsu. 2016.12.039

7. Malik R, Linos D (2016) Intraoperative neuromonitoring in thyroid surgery: a systematic review. World J Surg 40:2051-2058. doi:10. 1007/s00268-016-3594-y

8. Pisanu A, Porceddu G, Podda M et al (2014) Systematic review with meta-analysis of studies comparing intraoperative neuromonitoring of recurrent laryngeal nerves versus visualization alone during thyroidectomy. J Surg Res 188:152-161. doi:10.1016/ j.jss.2013.12.022

9. Lombardi CP, Carnassale G, Damiani G, et al (2016) "The final countdown": is intraoperative, intermittent neuromonitoring really useful in preventing permanent nerve palsy? Evidence from a metaanalysis. Surgery. doi: 10.1016/j.surg.2016.06.049

10. Higgins TS, Gupta R, Ketcham AS et al (2011) Recurrent laryngeal nerve monitoring versus identification alone on post-thyroidectomy true vocal fold palsy: a meta-analysis. Laryngoscope 121:10091017. doi:10.1002/lary. 21578

11. Sanabria A, Ramirez A, Kowalski LP et al (2013) Neuromonitoring in thyroidectomy: a meta-analysis of effectiveness from randomized controlled trials. Eur Arch Otorhinolaryngol 270:2175-2189. doi: 10.1007/s00405-013-2557-2

12. Yang S, Zhou L, Lu Z et al (2017) Systematic review with metaanalysis of intraoperative neuromonitoring during thyroidectomy. Int J Surg 39:104-113. doi:10.1016/j.ijsu.2017.01.086

13. Zheng S, Xu Z, Wei Y et al (2013) Effect of intraoperative neuromonitoring on recurrent laryngeal nerve palsy rates after thyroid surgery - a meta-analysis. J Formos Med Assoc 112:463-472. doi:10.1016/j.jfma.2012.03.003

14. Thomusch O, Sekulla C, Walls G et al (2002) Intraoperative neuromonitoring of surgery for benign goiter. Am J Surg 183: 673-678

15. Barczyński M, Konturek A, Stopa M et al (2011) Clinical value of intraoperative neuromonitoring of the recurrent laryngeal nerves in improving outcomes of surgery for well-differentiated thyroid cancer. Polish J Surg 83:196-203. doi:10.2478/v10035-011-0030-8

16. Rocke DJ, Goldstein DP, de Almeida JR (2016) A cost-utility analysis of recurrent laryngeal nerve monitoring in the setting of total thyroidectomy. JAMA Otolaryngol Neck Surg 142:1199. doi:10. 1001/jamaoto.2016.2860

17. Sanguinetti A, Parmeggiani D, Lucchini R et al (2014) Intraoperative recurrent laryngeal nerve monitoring in thyroid 
surgery evaluation of its use in terms of "spending review". Ann Ital Chir 85:418-421

18. Sturgeon C, Sturgeon T, Angelos P (2009) Neuromonitoring in thyroid surgery: attitudes, usage patterns, and predictors of use among endocrine surgeons. World J Surg 33:417-425. doi:10. 1007/s00268-008-9724-4

19. Barczyński M, Randolph GW, Cernea C, International Neural Monitoring Study Group in Thyroid and Parathyroid Surgery (2016) International survey on the identification and neural monitoring of the EBSLN during thyroidectomy. Laryngoscope 126: 285-291. doi:10.1002/lary.25548

20. Duclos A, Lifante J-C, Ducarroz S et al (2011) Influence of intraoperative neuromonitoring on surgeons' technique during thyroidectomy. World J Surg 35:773-778. doi:10.1007/s00268-011-0963-4

21. Jeannon J-P, Orabi AA, Bruch GA et al (2009) Diagnosis of recurrent laryngeal nerve palsy after thyroidectomy: a systematic review. Int J Clin Pract 63:624-629. doi:10.1111/j.1742-1241.2008.01875.x

22. Yarbrough DE, Thompson GB, Kasperbauer JL et al (2004) Intraoperative electromyographic monitoring of the recurrent laryngeal nerve in reoperative thyroid and parathyroid surgery. Surgery 136:1107-1115. doi:10.1016/j.surg.2004.06.040

23. Moher D, Liberati A, Tetzlaff J et al (2009) Preferred reporting items for systematic reviews and meta-analyses: the PRISMA statement. PLoS Med 6:e1000097. doi:10.1371/journal.pmed.1000097

24. Shea BJ, Grimshaw JM, Wells GA et al (2007) Development of AMSTAR: a measurement tool to assess the methodological quality of systematic reviews. BMC Med Res Methodol 7:10. doi:10.1186/ 1471-2288-7-10

25. Shea BJ, Bouter LM, Peterson J et al (2007) External validation of a measurement tool to assess systematic reviews (AMSTAR). PLoS One 2:e1350. doi:10.1371/journal.pone.0001350

26. Jadad AR, Cook DJ, Browman GP (1997) A guide to interpreting discordant systematic reviews. CMAJ 156:1411-1416

27. Rulli F, Ambrogi V, Dionigi G et al (2014) Meta-analysis of recurrent laryngeal nerve injury in thyroid surgery with or without intraoperative nerve monitoring. Acta Otorhinolaryngol Ital 34:223-229
28. Schneider R, Randolph GW, Sekulla C et al (2013) Continuous intraoperative vagus nerve stimulation for identification of imminent recurrent laryngeal nerve injury. Head Neck 35:1591-1598. doi:10.1002/hed.23187

29. Bergenfelz A, Jansson S, Kristoffersson A et al (2008) Complications to thyroid surgery: results as reported in a database from a multicenter audit comprising 3,660 patients. Langenbeck's Arch Surg 393:667-673. doi:10.1007/s00423-008-0366-7

30. Fontenot TE, Randolph GW, Setton TE et al (2015) Does intraoperative nerve monitoring reliably aid in staging of total thyroidectomies? Laryngoscope 125:2232-2235. doi:10.1002/lary.25133

31. Dralle H, Sekulla C, Lorenz K et al (2012) Loss of the nerve monitoring signal during bilateral thyroid surgery. Br J Surg 99:10891095. doi:10.1002/bjs.8831

32. Bergenfelz A, Salem AF, Jacobsson H et al (2016) Risk of recurrent laryngeal nerve palsy in patients undergoing thyroidectomy with and without intraoperative nerve monitoring. Br J Surg 103:18281838. doi:10.1002/bjs.10276

33. Chan W-F, Lang BH-H, Lo C-Y (2006) The role of intraoperative neuromonitoring of recurrent laryngeal nerve during thyroidectomy: a comparative study on 1000 nerves at risk. Surgery 140:866873. doi:10.1016/j.surg.2006.07.017

34. Wojtczak B, Kaliszewski K, Sutkowski K et al (2016) The learning curve for intraoperative neuromonitoring of the recurrent laryngeal nerve in thyroid surgery. Langenbeck's Arch Surg. doi:10.1007/ s00423-016-1438-8

35. Schneider R, Randolph GW, Barczynski M et al (2016) Continuous intraoperative neural monitoring of the recurrent nerves in thyroid surgery: a quantum leap in technology. Gland Surg 5:607-616. doi: 10.21037/gs.2016.11.10

36. Barczyński M, Konturek A, Pragacz K et al (2014) Intraoperative nerve monitoring can reduce prevalence of recurrent laryngeal nerve injury in thyroid reoperations: results of a retrospective cohort study. World J Surg 38:599-606. doi:10.1007/s00268-013-2260-x 